\title{
Avaliação de formulações de fosfito de potássio sobre Colletotrichum gloeosporioides in vitro e no controle pós- infeccional da mancha foliar de Glomerella em macieira
}

\author{
Leonardo Araújo ${ }^{1}$, Rosa M. Valdebenito-Sanhueza² \& Marciel J. Stadnik ${ }^{1}$ \\ ${ }^{1}$ Departamento de Fitotecnia, Centro de Ciências Agrárias, Universidade Federal de Santa Catarina, CEP 88040-900, \\ Florianópolis, SC, Brasil; ${ }^{2}$ Proterra Engenharia Agronômica, 95200-000, Vacaria, RS, Brasil
}

Autor para correspondência: Marciel J. Stadnik, e-mail: stadnik@cca.ufsc.br

\begin{abstract}
RESUMO
A eficiência de diferentes formulações comerciais de fosfitos de potássio foi avaliada no controle de Colletotrichum gloeosporioides em experimentos in vitro e in vivo. Nos testes in vitro, foram utilizadas doses semelhantes de íon fosfito, das formulações N-P-K 0-4020, 0-30-20 e 0-20-20, que foram incorporados ao meio de cultura BDA. Um disco de meio com o fungo foi transferido para cada placa de Petri e 7 dias após, avaliou-se o diâmetro da colônia. Mudas de macieira 'Gala' foram inoculadas com C. gloeosporioides e após 48 $\mathrm{h}$ pulverizadas com as doses de registro das seguintes formulações (p.c.) dos fertilizantes N-P-K: 0-40-20 (a:1,5 $\mu \mathrm{L} / \mathrm{mL}),(\mathrm{b}: 1,5 \mu \mathrm{L} / \mathrm{mL})$; 0-30-20 (c:1,5 $\mu \mathrm{L} / \mathrm{mL}),(\mathrm{d}: 2,5 \mu \mathrm{L} / \mathrm{mL}) ; 0-20-20(\mathrm{e}: 2 \mu \mathrm{L} / \mathrm{mL}),(\mathrm{f}: 3 \mu \mathrm{L} / \mathrm{mL})$; água (testemunha). Em outro ensaio, utilizaram-se somente fosfitos de potássio de formulação 0-40-20. Avaliou-se a percentagem de lesões 10 dias após a inoculação. Os fosfitos de formulação 040-20 (pH 3) interferiram no desenvolvimento do C. gloeosporioides, causando diminuição de $94 \%$ no crescimento micelial das colônias. Quando pulverizado $48 \mathrm{~h}$ após a inoculação, somente uma formulação de fosfito $0-40-20$ reduziu significativamente a severidade da mancha foliar de Glomerella, isto é, em $62 \%$.
\end{abstract}

Palavras-chave: Malus domestica, Colletotrichum gloeosporioides, fosfito de potássio, mancha foliar de Glomerella.

\begin{abstract}
Evaluation of potassium phosphite formulations against Colletotrichum gloeosporioides in vitro and for post-infection control of Glomerella leaf spot in apple

The efficiency of different commercial formulations of potassium phosphite on Colletotrichum gloeosporioides was evaluated in vitro and in vivo. In in vitro tests similar doses of phosphite ion in N-P-K formulations $0-40-20,0-30-20$ and 0-20-20 were incorporated into PDA medium in Petri dishes. A disk of the medium with the fungus was transferred to each Petri dish and colony diameter was measured after 7 days. Plants of apple 'Gala' were inoculated with $C$. gloeosporioides and after $48 \mathrm{~h}$ were sprayed with recommended doses of the following formulations (p.c.) of N-P-K fertilizers: 0-40-20 (a:1.5 $\mu \mathrm{L} / \mathrm{mL}),(\mathrm{b}: 1.5 \mu \mathrm{L} / \mathrm{mL}) ; 0-30-20(\mathrm{c}: 1.5 \mu \mathrm{L} / \mathrm{mL}),(\mathrm{d}: 2.5 \mu \mathrm{L} /$ $\mathrm{mL}) ; 0-20-20(\mathrm{e}: 2 \mu \mathrm{L} / \mathrm{mL}),(\mathrm{f}: 3 \mu \mathrm{L} / \mathrm{mL})$, water (control). In a second experiment, only potassium phosphite in the formulation 0-40-20 was used. Disease severity was evaluated 10 days after inoculation. The phosphites in the formulation 0-40-20 (pH 3) interferred with mycelial development of $C$. gloeosporioides and reduced colony diameter by $94 \%$. In plants sprayed at $48 \mathrm{~h}$ after inoculation, only one formulation of phosphite (0-40-20) significantly reduced the severity of Glomerella leaf spot and did so by $62 \%$.
\end{abstract}

Keywords: Malus domestica, Colletotrichum gloeosporioides, potassium phosphites, Glomerella leaf spot.

A mancha foliar da Glomerella (MFG) foi observada pela primeira vez em 1983 em pomares de macieira (Malus domestica Bork) (Leite et al., 1988). Desde então, a freqüência da doença vem aumentando, sendo que atualmente ocorre em praticamente todas as regiões produtoras de maçãs no Brasil (Boneti et al., 2004). A MFG manifesta-se principalmente no verão, quando pode provocar desfolhamento superior a $75 \%$ e como conseqüência, reduzir drasticamente a produção das plantas no ano seguinte (Epagri, 2002). Os sintomas iniciais da doença são manchas vermelho-arroxeadas, já visíveis 48 $\mathrm{h}$ após o inicio da infecção e que evoluem para manchas necróticas. As folhas lesionadas amarelecem e caem precocemente, entre oito a dez dias (Crusius et al., 2002). A MFG é causada por Colletotrichum gloeosporioides (Penz.) Penz. \& Sacc e afeta as cultivares de macieiras descendentes da 'Golden Delicious'. Desta maneira, a 'Gala' principal cultivar plantada no Brasil apresenta alta suscetibilidade, enquanto a 'Fuji', outra cultivar importante, não é afetada (Epagri, 2002).

A pulverização com fungicidas de ação preventiva a cada cinco ou dez dias, ou ainda, quando houver acúmulo de $30 \mathrm{~mm}$ de chuvas nos intervalos, é o método mais utilizado para o controle da MFG. Contudo, mesmo com proteção química permanente pode haver perda de controle 
quando a pressão de inóculo é muito alta (Boneti et al., 2004). Sistemas de Alerta para a doença já vêm sendo estudados (Boneti et al., 2004), mas as vantagens destes são diminuídas pelo fato de não se contar com produtos com ação curativa. Dentro do contexto do manejo integrado e baixa pressão de inóculo, fertilizantes à base de fosfitos vêm sendo empregados no controle de doenças de plantas frutíferas como o oídio (Geelen, 1999) e a sarna (Boneti \& Katsurayama, 2005) em macieira, a podridão-do-pé (Dianese et al., 2007) e a varíola (Dianese et al., 2008) em mamoeiro, além da mancha de Phoma em cafeeiro (Nojosa et al., 2009). Segundo Cohen \& Coffey (1986), os fosfitos têm acidez característica, já que seu íon $\left(\mathrm{HPO}_{3}^{2-}\right)$ é oriundo do ácido fosforoso $\left(\mathrm{H}_{3} \mathrm{PO}_{3}\right)$, sendo compostos não fitotóxicos e apresentando atividade fungicida na planta. O fosfito é translocado via floema e xilema e trata-se de uma forma não metabolizável de fósforo que é absorvida pelas folhas, podendo se acumular na planta por até 150 dias (Guest \& Grant, 1991; Malusa \& Tosi, 2005).

Existem controvérsias sobre o modo de ação dos fosfitos, devido alguns trabalhos comprovarem que estes sais podem atuar diretamente sobre o patógeno (Fenn \& Coffey, 1984; Ribeiro et al., 2006) e/ou pela indução de resistência (Saindrenant et al., 1988). Em macieira, Araújo et al. (2008) descreveram efeito curativo de um fosfito de potássio 0-40-20 pulverizado $24 \mathrm{~h}$ após infecção, reduzindo a severidade da MFG em 90 \%. Já aplicações preventivas do produto, não diminuíram a severidade dessa doença. Entretanto, o fosfito pulverizado sete dias antes da inoculação em condições de casa-de-vegetação controla a sarna da macieira (Boneti \& Katsurayama, 2005). No entanto, diversos trabalhos têm mostrado que marcas e/ou formulações de fosfitos podem apresentar eficiência diferente no controle de doenças (Sonego et al., 2003; Brackman et al., 2005; Dianese et al., 2007). Apesar disso e do seu uso intensivo na pomicultura brasileira, inexiste atualmente uma avaliação comparativa da eficiência dos diferentes fosfitos utilizados no controle da MFG.

Desta forma, o objetivo do trabalho foi avaliar o efeito de diferentes marcas e/ou formulações de fosfitos de potássio sobre C. gloeosporioides in vitro e em ensaios com tratamentos pós-infeccional em mudas de macieiras no controle da MFG.

Os experimentos foram realizados na Estação Experimental de Fruticultura Temperada da Embrapa Uva e Vinho e utilizaram-se os seguintes fosfitos: (a) 40-20: $0 \%(\mathrm{~N})-40 \%\left(\mathrm{P}_{2} \mathrm{O}_{5}\right)-20 \%\left(\mathrm{~K}_{2} \mathrm{O}\right), 150 \mathrm{~mL} / 100 \mathrm{~L}$; (b) $40-20$, $150 \mathrm{~mL} / 100 \mathrm{~L}$; (c) 30-20: $0 \%(\mathrm{~N})-30 \%\left(\mathrm{P}_{2} \mathrm{O}_{5}\right)-20 \%\left(\mathrm{~K}_{2} \mathrm{O}\right)$, $150 \mathrm{~mL} / 100 \mathrm{~L}$; (d) $30-20,250 \mathrm{~mL} / 100 \mathrm{~L}$; (e) $20-20$ : $0 \%(\mathrm{~N})-20 \%\left(\mathrm{P}_{2} \mathrm{O}_{5}\right)-20 \%\left(\mathrm{~K}_{2} \mathrm{O}\right), 200 \mathrm{~mL} / 100 \mathrm{~L}$; e (f) $20-20$, $300 \mathrm{~mL} / 100 \mathrm{~L}$. Mediu-se o $\mathrm{pH}$ dos fosfitos de potássio com o auxílio de um pH-metro portátil (Marca: Schott - Alemanha), com doses semelhantes de íon fosfito, presente nas diferentes formulações líquidas 0-40-20: $(1,5 \mu \mathrm{L} / \mathrm{mL}) ; 0-30-20:(2,5 \mu \mathrm{L} / \mathrm{mL}) ; 0-20-20:(3,0 \mu \mathrm{L} /$ $\mathrm{mL}$ ). Mediu-se, também o $\mathrm{pH}$ da água destilada, utilizada como testemunha.
Em todos os experimentos foi usado o isolado CGF1 de C. gloeosporioides da coleção da Embrapa Uva e Vinho, proveniente de plantas infectadas da cultivar Gala. Para obtenção de inóculo foram usadas culturas desenvolvidas em BDA por dez dias a $25^{\circ} \mathrm{C}$ e $12 \mathrm{~h}$ de fotoperíodo. As colônias formadas foram raspadas superficialmente com espátula de Drigalsky e a concentração da suspensão de conídios foi ajustada com o auxílio da câmara de Neubauer, conforme especificado em cada experimento.

Para determinar o efeito in vitro sobre $C$. gloeosporioides foram incorporadas doses semelhantes de íon fosfito das diferentes formulações em meio BDA a $45^{\circ} \mathrm{C}$, imediatamente antes de vertê-lo. Verteu-se $15 \mathrm{~mL}$ de meio BDA em cada placa de $10 \mathrm{~cm}$ de diâmetro e testaram-se os seguintes tratamentos: (a) 40-20 $(1,5 \mu \mathrm{L} / \mathrm{mL})$; (b) $40-20$ $(1,5 \mu \mathrm{L} / \mathrm{mL})$; (c) $30-20(2,5 \mu \mathrm{L} / \mathrm{mL})$; (d) $30-20(2,5 \mu \mathrm{L} / \mathrm{mL})$; (e) $20-20(3 \mu \mathrm{L} / \mathrm{mL})$; (f) $20-20(3,0 \mu \mathrm{L} / \mathrm{mL})$; Testemunha (somente BDA). Para avaliar o efeito dos produtos sobre o crescimento de colônias de C. gloeosporioides, após a solidificação do meio, um disco de $9 \mathrm{~mm}$ de diâmetro contendo micélio do fungo foi transferido para o centro de cada placa, e as culturas incubadas a $24^{\circ} \mathrm{C}$ e $12 \mathrm{~h}$ de fotoperíodo. Diâmetros perpendiculares das colônias foram medidos diariamente aos três a, sete dias após o inicio da incubação, com o auxílio de um paquímetro. Com os dados foi calculado o IVCM (Índice de Velocidade de Crescimento Micelial) expresso $\mathrm{em} \mathrm{mm} /$ dia, utilizando-se para tanto a fórmula (Araújo et al., 2008): IVCM $=\sum$ (D - Da) / N, onde: D: Diâmetro médio atual; Da: Diâmetro médio anterior; N: número de dias após a repicagem.

Para avaliar o efeito pós-infeccional dos fosfitos de potássio no controle da MFG em mudas de macieira foram realizados dois ensaios. No ensaio 1 foram testados todos os fosfitos descritos anteriormente, enquanto que no ensaio 2 foram utilizados somente os fosfitos que controlaram o C. gloeosporioides in vitro e in vivo. Para tanto, mudas de macieira foram cultivadas em casa de vegetação em vasos plásticos contendo como substrato solo argiloso. No ensaio 1 , as plantas com um ano de idade, foram mantidas em vasos de dois litros, enquanto que no ensaio 2, as plantas com dois anos de idade, foram cultivadas em vasos de seis litros. Todas as plantas foram da cv. Gala enxertada sobre a porta-enxerto EM7. Plantas foram irrigadas manualmente de acordo com as suas necessidades hídricas. Nos dois ensaios, $24 \mathrm{~h}$ antes da inoculação, as plantas foram incubadas com $100 \%$ de umidade, para se ter uma maior abertura dos estômatos. Findo este período, as mudas foram inoculadas com uma suspensão de $3 \times 10^{5}$ conídios $/ \mathrm{mL}$ e mantidas no escuro a $100 \%$ UR e $22^{\circ} \mathrm{C}$ por $48 \mathrm{~h}$.

No ensaio 1, mudas foram tratadas às $48 \mathrm{~h}$ após a inoculação (hai) com as doses de registro dos fosfitos: (a) $40-20(1,5 \mu \mathrm{L} / \mathrm{mL})$; (b) $40-20(1,5 \mu \mathrm{L} / \mathrm{mL})$; (c) $30-20$ $(1,5 \mu \mathrm{L} / \mathrm{mL})$; (d) $30-20(2,5 \mu \mathrm{L} / \mathrm{mL})$; (e) $20-20(2,0 \mu \mathrm{L} / \mathrm{mL})$; (f) $20-20(3,0 \mu \mathrm{L} / \mathrm{mL})$ e água destilada. As plantas tratadas permaneceram em bancadas da casa de vegetação até a avaliação da severidade da MFG, estimando-se visualmente 
a porcentagem de tecido necrosado (Araújo et al., 2008) de quinze folhas de um ramo aos cinco, seis, sete, oito e dez dias após a inoculação (dai). Com os dados, calculou-se área abaixo da curva do progresso da doença (AACPD), usandose a seguinte fórmula: $\mathrm{AACPD}=[((\mathrm{y} 1+\mathrm{y} 2) / 2 *(\mathrm{t} 2-\mathrm{t} 1)]$, onde y1 e y2 são duas avaliações consecutivas de severidade realizadas nos tempos $\mathrm{t} 1$ e $\mathrm{t} 2$, respectivamente. No ensaio 2 , mudas foram pulverizadas às 48 hai, com os fosfitos (a) 40-20 $(1,5 \mu \mathrm{L} / \mathrm{mL})$, (b) $40-20(1,5 \mu \mathrm{L} / \mathrm{mL})$ e água destilada, além disso, avaliou-se a severidade da MFG conforme descrito anteriormente.

O delineamento usado nos experimentos foi o completamente casualizado, com cinco repetições nos testes in vitro e três repetições nos ensaios in vivo. Os dados dos experimentos foram submetidos à análise de variância, seguido do teste de separação de médias $\operatorname{SNK}(\alpha \leq 0,05)$, com o software Statistica 6,0 - Stat Soft ${ }^{\circledR}$, versão 2001.

Doses crescentes de fosfitos nas formulações resultaram em $\mathrm{pH}$ mais baixo. Em doses semelhantes de íon fosfito, o $\mathrm{pH}$ dos fosfitos das formulações 0-40-20, 0-30-20 e 0-20-20 foram em média 3,0, 4,1 e 6,8, respectivamente (Tabela 1). Todos os fosfitos testados reduziram o diâmetro das colônias e a velocidade de crescimento micelial de $C$. gloeosporioides em relação à testemunha. As formulações 0-40-20, 0-30-20, 0-20-20 reduziram em média 94, 85 e $36 \%$ o diâmetro das colônias e IVCM (Tabela 1).

No ensaio 1, quando se utilizou diferentes formulações de fosfitos de potássio, as plantas testemunhas apresentaram $9 \%$ de severidade e 33 de AACPD. O fosfito (b) 40-20 aplicado curativamente às 48 hai reduziu em $62 \%$ a área foliar necrosada aos 10 dai e AACPD em mudas de macieira. Os demais tratamentos não diferiram da testemunha em nenhuma das variáveis analisadas (Figura 1). No ensaio 2, quando mudas de macieira foram pulverizadas somente com fosfitos de formulação 0-4020 , as testemunhas apresentaram $18 \%$ de severidade e 60 de AACPD. Nesse ensaio, o fosfito de potássio (b) 40-20 reduziu significativamente em $40 \%$ a área foliar necrosada aos 10 dai e $37 \%$ para $\mathrm{AACPD}$, em relação à testemunha (Figura 2).

A maior parte dos fungos tolera uma ampla faixa de $\mathrm{pH}$, porém existe uma faixa ótima de crescimento que varia de 5,0 a 6,5. Assim, microorganismos que se encontram em meios com pHs abaixo ou acima da faixa ideal, terão um desenvolvimento prejudicado. Liu et al. (2005), variando pHs (3 a 10) in vitro, demonstraram que o C. gloeosporioides, agente causal da antracnose da lichia, apresenta maior crescimento micelial entre pH 5,0 a 6,0 e maior germinação de conídios entre $\mathrm{pH} 6,0$ a 7,0. No presente trabalho fosfitos de formulação 0-40-20 tinham $\mathrm{pH} 3,0$, enquanto os 0-30-20 em torno de 4,0 e os 0-20-20 tinham um pH neutro (Tabela 1). Esta diferença de $\mathrm{pH}$ nos fosfitos com quantidades equivalentes de íon fosfito nas formulações pode ser devido a um desequilíbrio na quantidade óxido de potássio $\left(\mathrm{K}_{2} \mathrm{O}\right)$ no meio (20\% nas formulações), quando se ajustou $\mathrm{HPO}_{3}$ nas diferentes formulações. Pois, o $\mathrm{K}_{2} \mathrm{O}$, dissociado em água forma o hidróxido de potássio $(2 \mathrm{KOH})$, que é uma base forte e deve ter aumentado o $\mathrm{pH}$ dos fosfitos de formulação 30-20 e 20-20, no ajuste do $\mathrm{P}_{2} \mathrm{O}_{4}$

Os resultados dos experimentos in vitro demonstraram atividade direta dos fosfitos de potássio sobre o crescimento micelial (Tabela 1). Em doses semelhantes, a ação dos fosfitos in vitro foi menor nas formulações com $\mathrm{pH}$ mais elevado, sugerindo que a acidez do fertilizante possa interferir diretamente no desenvolvimento do fungo. Resultados semelhantes foram observados por Araújo et al. (2008), comparando concentrações de fosfito de potássio 040-20, em pH 2,0 e pH 7,0. Na maior concentração testada $(0,5 \mu \mathrm{L} / \mathrm{mL})$ houve uma inibição total do crescimento micelial do fungo, em relação à testemunha, em $\mathrm{pH} 2,0$. Por outro lado, em pH 7,0, nesta mesma concentração, a redução do crescimento micelial do C. gloeosporioides, foi de $50 \%$. $\mathrm{O}$ efeito direto de fosfitos de potássio sobre a germinação de conídios (Ribeiro et al., 2006) e o crescimento micelial

TABELA 1 - Efeito das formulações e marcas de fosfito incorporado ao meio BDA, sobre o diâmetro da colônia e índice de velocidade de crescimento micelial (IVCM) de Colletotrichum gloeosporioides. Valor de pH dos fosfitos de potássio em meio BDA com doses semelhantes de íon fosfito, presente nas diferentes formulações de N-P-K: 0-40-20, $(1.5 \mu \mathrm{L} / \mathrm{mL})$; 0-30-20, $(2.5 \mu \mathrm{L} / \mathrm{mL}) ; 0-20-20,(3 \mu \mathrm{L} / \mathrm{mL})$. Na análise estatística os dados foram transformados para raiz (x). Colunas com mesma letra não diferem entre si pelo teste de SNK $(\alpha \geq 0,05)$

\begin{tabular}{lccc}
\hline \hline Tratamentos & $\mathbf{p H}$ & $\begin{array}{c}\text { Diâmetro colônia (mm) } \\
\text { 7 Dias a pós a repicagem }\end{array}$ & IVCM \\
\hline (a) $40-20$ & 3,0 & $3,5 \mathrm{a}$ & $0,50 \mathrm{a}$ \\
(b) $40-20$ & 3,0 & $3,8 \mathrm{a}$ & $0,55 \mathrm{a}$ \\
(c) $30-20$ & 3,8 & $10,7 \mathrm{~b}$ & $1,53 \mathrm{~b}$ \\
(d) $30-20$ & 4,4 & $8,9 \mathrm{~b}$ & $1,26 \mathrm{~b}$ \\
(e) $20-20$ & 6,6 & $37,9 \mathrm{c}$ & $5,38 \mathrm{c}$ \\
(f) $20-20$ & 7,0 & $42,5 \mathrm{~d}$ & $6,07 \mathrm{~d}$ \\
Testemunha & 6,5 & $63,0 \mathrm{e}$ & $9,01 \mathrm{e}$ \\
\hline CV \%: & & 6,4 & 6,4 \\
\hline
\end{tabular}




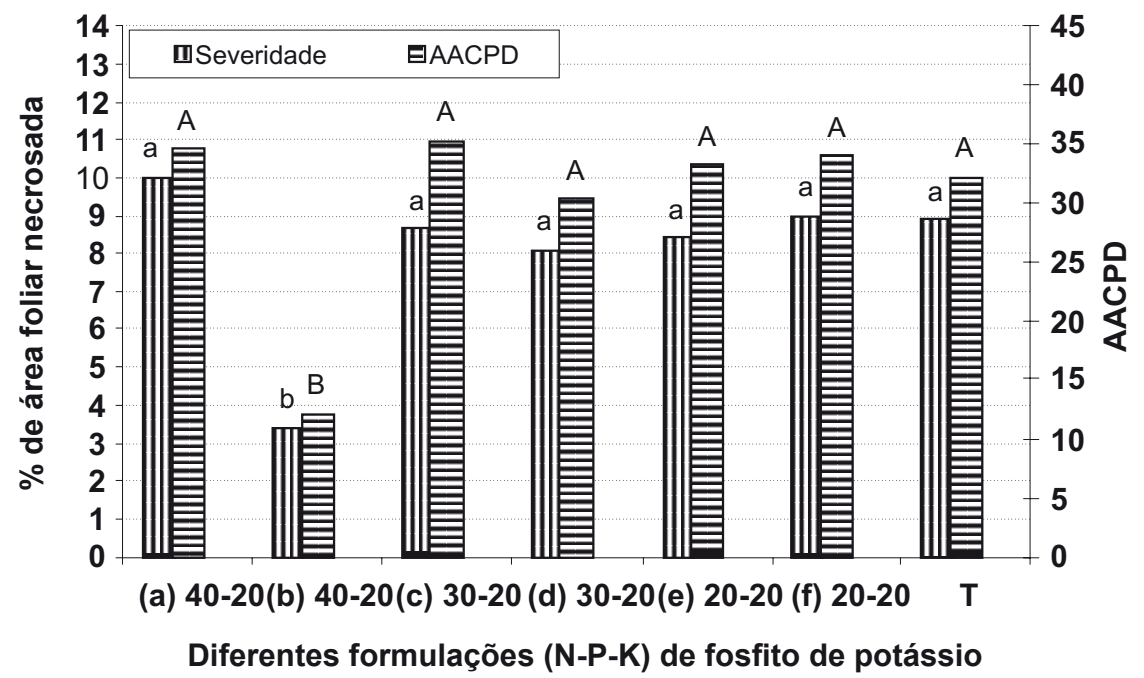

FIGURA 1 - Efeito do tratamento pós-infeccional (48 h após a inoculação), com diferentes formulações e doses comerciais de fosfitos de potássio, sobre a percentagem de área foliar necrosada aos 10 dias após a inoculação (Colletotrichum gloeosporioides) e área abaixo da curva de progresso da doença (AACPD), em mudas de macieira 'Gala'. $\mathrm{Na}$ análise estatística os dados foram transformados para raiz (x). Colunas com mesmas letras minúsculas e maiúsculas indicam que não há diferença estatística pelo teste de SNK $(\alpha \geq 0,05)$. T: Testemunha. Coeficiente variação para ( $\%$ área foliar necrosada): $13,6 \%$ e (AACPD): $12,2 \%$.

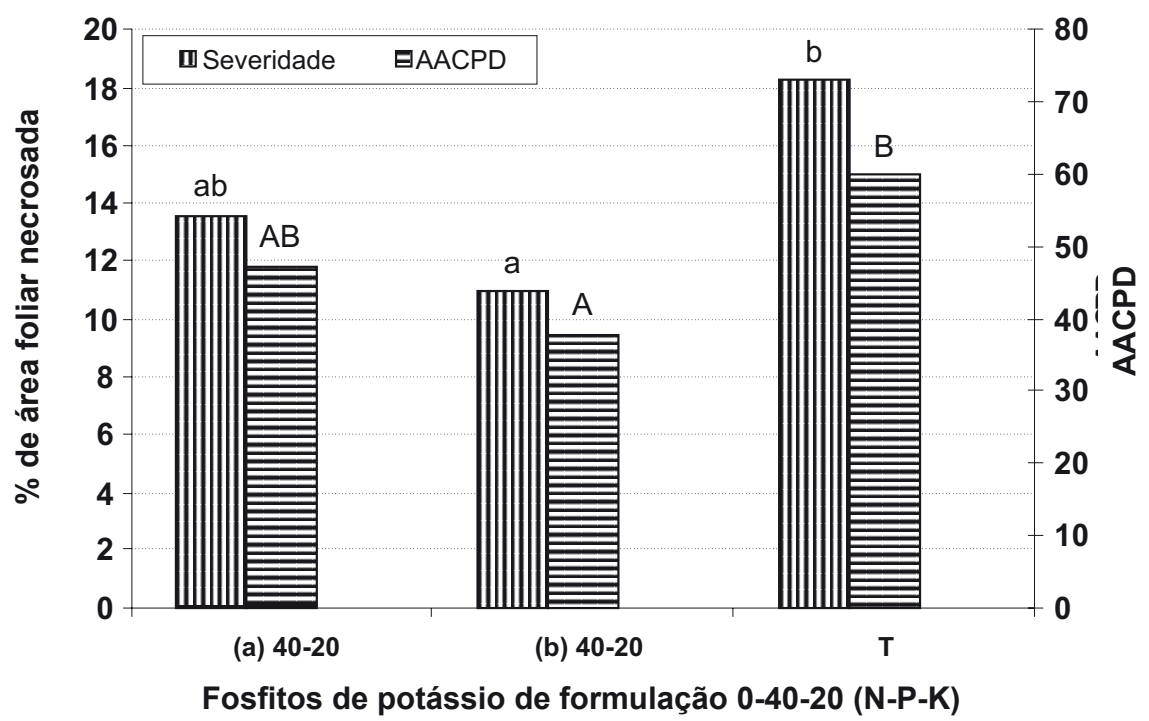

FIGURA 2 - Efeito do tratamento pós-infeccional (48h após a inoculação), com a formulação 0-40-20 (N-P-K) de fosfitos de potássio sobre a percentagem de área foliar necrosada aos 10 dias após a inoculação (Colletotrichum gloeosporioides) e área abaixo da curva de progresso da doença (AACPD) em mudas de macieira 'Gala'. Na análise estatística os dados foram transformados para raiz (x). Colunas com mesmas letras minúsculas e maiúsculas indicam que não há diferença estatística pelo teste de SNK $(\alpha \geq 0,08)$. T:Testemunha apenas água. Coeficiente variação para (\% área foliar necrosada): $12,6 \%$ e (AACPD): $11,8 \%$. 
de fungos (Nojosa et al., 2009) tem sido verificado em outros patossistemas.

Os fosfitos testados in vivo apresentaram eficiência diferente no controle da MFG. A formulação (b) 40-20 aplicada às 48 hai reduziu em até $62 \%$ a severidade da MFG (Figuras 1 e 2). Estes dados reforçam os resultados encontrados por Araújo et al. (2008), que relataram efeito curativo do fosfito 0-40-20 sobre a MFG até 24 hai. Também, Dianese et al. (2007), que testaram diferentes formulações de fosfito para o controle da podridão-do-pé em mamoeiro, causada por Phytophthora palmivora Butler obtiveram os melhores resultados para uma formulação 0-40-20. Diferentemente dos resultados agora relatados, Brackman et al. (2005) testaram diferentes formulações de fosfitos, aplicadas em maçãs antes do armazenamento em câmara, e encontraram a melhor eficiência na formulação 0-2826 para o controle de podridões de pós-colheita. Algumas das hipóteses que podem ser discutidas para explicar os resultados dos fosfitos in vivo neste trabalho são: a) os fosfitos têm baixo efeito protetor sobre $C$. gloeosporioides porque são rapidamente absorvidos pelas folhas (Guest \& Grant, 1991; Malusa \& Tosi, 2005) e afetariam os conídios somente por um período reduzido. Entretanto, é provável que o potencial deles para inibição do micélio se expresse pela acumulação desses produtos nos ápices de crescimento (Guest \& Grant, 1991; Malusa \& Tosi, 2005), tecidos mais suscetíveis (Araújo et al., 2008), durante a fase de sua colonização; b) os fosfitos, tendo um efeito fungistático transiente, desfavoreceriam o desenvolvimento do fungo e o deixariam exposto por mais tempo à radiação UV, dessecação, temperaturas desfavoráveis e ação de antagonistas.

Tanto nos ensaios in vitro como in vivo obteve-se os melhores resultados no controle de C. gloeosporioides com os fosfitos $0-40-20$, que possuíam o menor $\mathrm{pH}(3,0)$ entre as formulações testadas. Howard et al. (1998), em ensaios com adubações foliares com duas fontes de potássio $\left(\mathrm{KNO}_{3}\right.$ e $\left.\mathrm{K}_{2} \mathrm{SO}_{4}\right)$ e diferentes valores de $\mathrm{pHs}(4,0$ e 6,0$)$ concluíram que plantas de algodão absorveram $10 \%$ a mais de potássio nas soluções mais ácidas. Também Rosolem et al. (2007) encontraram uma melhor absorção de nutrientes de adubos foliares em soluções ácidas. Desta forma, no presente trabalho o baixo $\mathrm{pH}$ (3) da formulação 0-40-20 pode ter aumentado a absorção do íon fosfito e/ou potássio interferindo no desenvolvimento do C. gloeosporioides.

Ainda nos ensaios in vivo, observou-se que fosfitos de mesma formulação e pH similares, não manifestaram a mesma eficiência no controle do C. gloeosporioides (Figuras 1 e 2 ). Resultados semelhantes foram obtidos por Brackman et al. (2005) que testaram cinco fosfitos de uma mesma formulação, obtendo diferentes efeitos. Além disso, Dianese et al. (2007) testaram sete fosfitos para o controle da podridão-do-pé em mamoeiro, obtendo resultados satisfatórios com apenas uma marca de uma formulação. Algumas das possíveis hipóteses que podem explicar estes diferentes comportamentos para fosfitos de mesma formulação seriam: excesso ou deficiência de algum elemento na solução, e/ou algum produto possa ser absorvido mais rapidamente que outro.

Desta forma, os resultados deste estudo sugerem que somente a formulação 0-40-20 reduz a severidade da MFG, e que as características ácidas destes fosfitos de potássio ( $\mathrm{pH} 3$ ) inibem o desenvolvimento do C. gloeosporioides, além de um provável favorecimento na absorção do íon fosfito e/ou potássio pelas plantas.

\section{AGRADECIMENTOS}

Os autores agradecem à Fundação de Apoio à Pesquisa Científica e Tecnológica do Estado de Santa Catarina - FAPESC, ao Conselho Nacional de Desenvolvimento Científico e Tecnológico - CNPq, e ao Programa Iberoamericano de Ciência e Tecnologia para o Desenvolvimento - CYTED pelo suporte financeiro.

\section{REFERÊNCIAS BIBLIOGRÁFICAS}

Araújo L, Borsato LC, Valdebenito-Sanhueza RM, Stadnik MJ (2008) Fosfito de potássio e ulvana no controle da mancha foliar da gala em macieira. Tropical Plant Pathology 33:74-80.

Boneti JIS, Becker WF, Katsurayama Y (2004) Sistema de previsão da mancha-foliar-da-gala em macieira, cultivar gala. Agropecuária Catarinense 17:64-67.

Boneti JIS, Katsurayama Y (2005) Viabilidade do uso de fosfitos no controle da sarna-da-macieira. Agropecuária Catarinense 18:51-54.

Brackmann A, Giehl RFH, Sestari I, Steffens CA, Faulin GDC, Pinto JAV (2005) Controle de podridão pós-colheita de Penicillium spp. em maçã 'fuji' com fosfitos e fungicidas. Revista Brasileira de Agrociência 11:251-254.

Cohen MD, Coffey MD (1986) Systemic fungicides and the control of oomycetes. Annual Review of Phytopathology 24:311-338.

Crusius LU, Forcelini CA, Sanhueza RMV, Fernandes JMC (2002) Epidemiology of apple leaf spot. Fitopatologia Brasileira 27:65-70.

Dianese AC, Blum LEB, Dutra JB, Lopes LF, Sena MC, Freitas LF, Yamanishi OK (2007) Redução da Podridão do Pé (Phytophthora palmivora) do Mamoeiro (Carica papaya) por Fosfitos. Fitopatologia Brasileira 32:166.

Dianese AC, Blum LEB, Dutra JB, Lopes LF, Sena MC, Freitas LF (2008) Avaliação do efeito de fosfitos na redução da varíola (Asperisporium caricae) do mamoeiro (Carica papaya). Revista Brasileira de Fruticultura 30:834-837.

Epagri (2002) A cultura da macieira. Florianópolis SC. Epagri.

Fenn ME, Coffey MD (1984) Studies on the in vitro and in vivo antifungal activity of Fosetyl-Al and Phosphorous acid. Phytopathology 74:606-611.

Geelen JA (1999) An evaluation of Agri-Fos Supra 400 for the control of black spot and powdery mildew of apple in Hawke's Bay. New York NY. Independent Horticultural Consultants. 
Guest DI, Grant BR (1991) The complex action of phosphonate antifungal agents. Biological Review 66:59-187.

Howard DD, Gwathmey CO, Sams CE (1998) Foliar feeding of cotton: evaluating potassium sources, potassium solution buffering, and boron. Agronomy Journal 90:740-746.

Leite Junior RP, Tsuneta M, Kishina AY (1988) Ocorrência da mancha foliar de Glomerella em macieira no estado do Paraná. Londrina PR. Instituto Agronômico do Paraná. Informe de Pesquisa no. 81 .

Liu A, Chen W, Li X (2005) Changes in the postharvest physiology and lychee fruits latently infected by anthracnose fungus and the biological characteristic of the pathogenic fungus of the disease. Acta Horticulturae 665:365-371.

Malusa E, Tosi L (2005) Phosphorous acid residues in apples after foliar fertilization: results of field trials. Food Additives and Contaminants 22:541-548.
Nojosa GBA, Resende MLV, Barguil BM, Moraes SRG, Vilas Boas CH (2009) Efeito de indutores de resistência em cafeeiro contra a mancha de Phoma. Summa Phytopathologica 35:60-62.

Ribeiro PMJ, Resende MLV, Pereira RB, Cavalcanti FR, Amaral DR, Pádua MA (2006) Fosfito de potássio na indução de resistência a Verticillium dahliae Kleb. em mudas de cacaueiro (Theobroma cacao L.). Ciência e Agrotecnologia 30:629-636.

Rosolem CA, Witacker JPT (2007) Adubação foliar com nitrato de potássio em algodoeiro. Bragantia 66:147-155.

Saindrenant P, Barchietto T, Avelino PJ, Bompeix G (1988) Effect of phosphite on phytoalexin accumulation in leaves of cowpea infected with Phytophthora cryptogea. Physiological and Molecular Plant Pathology 32:425-435.

Sonego OR, Garrido LR, Czermainski ABC (2003) Avaliação de fosfitos no controle do míldio da videira. Bento Gonçalves RS. Embrapa Uva e Vinho. Boletim de Pesquisa e Desenvolvimento no. 11 .

TPP 9051 - Recebido 13 Abril 2009 - Aceito 22 Janeiro 2010 Editor de Seção: John C. Sutton 